\title{
Communication
}

\section{Endosomal Escape and Delivery of CRISPR/Cas9 Genome Editing Machinery Enabled by Nanoscale Zeolitic Imidazolate Framework}

Shahad K. Alsaiari, Sachin Patil, Mram Alyami, Kholod Alamoudi, fajr Aleisa, Jasmeen Merzaban, Mo Li, and Niveen M Khashab

J. Am. Chem. Soc., Just Accepted Manuscript • DOI: 10.1021/jacs.7b11754 • Publication Date (Web): 22 Dec 2017

Downloaded from http://pubs.acs.org on December 26, 2017

\section{Just Accepted}

"Just Accepted" manuscripts have been peer-reviewed and accepted for publication. They are posted online prior to technical editing, formatting for publication and author proofing. The American Chemical Society provides "Just Accepted" as a free service to the research community to expedite the dissemination of scientific material as soon as possible after acceptance. "Just Accepted" manuscripts appear in full in PDF format accompanied by an HTML abstract. "Just Accepted" manuscripts have been fully peer reviewed, but should not be considered the official version of record. They are accessible to all readers and citable by the Digital Object Identifier (DOI®). "Just Accepted" is an optional service offered to authors. Therefore, the "Just Accepted" Web site may not include all articles that will be published in the journal. After a manuscript is technically edited and formatted, it will be removed from the "Just Accepted" Web site and published as an ASAP article. Note that technical editing may introduce minor changes to the manuscript text and/or graphics which could affect content, and all legal disclaimers and ethical guidelines that apply to the journal pertain. ACS cannot be held responsible for errors or consequences arising from the use of information contained in these "Just Accepted" manuscripts. 


\title{
Endosomal Escape and Delivery of CRISPR/Cas9 Genome Ed- iting Machinery Enabled by Nanoscale Zeolitic Imidazolate Framework
}

\author{
Shahad K. Alsaiari, ${ }^{\dagger}$ Sachin Patil, ${ }^{\dagger}$ Mram Alyami, ${ }^{\dagger}$ Kholod O. Alamoudi, ${ }^{\dagger}$ Fajr A. Aleisa, ${ }^{\S}$ Jasmeen \\ S. Merzaban, ${ }^{\S}$ Mo Li, ${ }^{\star}$ Niveen M. Khashab ${ }^{*} \dagger$ \\ ${ }^{\dagger}$ Smart Hybrid Materials (SHMs) Laboratory, Advanced Membranes and Porous Materials Center, King Abdullah \\ University of Science and Technology (KAUST), Thuwal 23955-690o, Saudi Arabia.
}

${ }^{\S}$ Cell Migration and Signaling Laboratory, Division of Biological and Environmental Science and Engineering, King Abdullah University of Science and Technology (KAUST), Thuwal 23955-6900, Saudi Arabia

\$Stem Cell and Regeneration Laboratory, Division of Biological and Environmental Science and Engineering, King Abdullah University of Science and Technology (KAUST), Thuwal 23955-690o, Saudi Arabia.

\section{Supporting Information}

\begin{abstract}
CRISPR/Cas9 is a combined protein (Cas9) and an engineered single guide RNA (sgRNA) genome editing platform that offers revolutionary solutions to genetic diseases. It has, however, a double delivery problem owning to the large protein size and the highly charged RNA component. In this work, we report the first example of CRISPR/Cas9 encapsulated by nanoscale zeolitic imidazole frameworks (ZIFs) with a loading efficiency of $17 \%$ and enhanced endosomal escape promoted by the protonated imidazole moieties. The gene editing potential of CRISPR/Cas9 encapsulated by ZIF-8 (CC-ZIFs) is further verified by knocking down the gene expression of green fluorescent protein by $37 \%$ over 4 days. The nanoscale CC-ZIFs are biocompatible and easily scaled-up offering excellent loading capacity and controlled co-delivery of intact Cas9 protein and sgRNA.
\end{abstract}

CRISPR/Cas9, the clustered regularly interspaced short palindromic repeat (CRISPR) associated proteins 9 (Cas9) technology, is a recently developed site-specific gene editing platform that uses single guide RNA (sgRNA) for site recognition. ${ }^{1-3}$ The protein complex targets the DNA sequence of interest via the sgRNA. DNA strands complementary to the sgRNA are cleaved by Cas9 protein. The break is then repaired by non-homologous end-joining (NHEJ) or homologydirected repair (HR). Recently, several studies have reported the applicability of CRISPR/Cas9 technology as a powerful therapeutic strategy against viruses, ${ }^{4}$ bacteria $^{5}$ and cancer. ${ }^{6}$ However, for all the enthusiastic upside surrounding the prospects of RNA-based therapeutics, there continues to be an equal and opposite downside that started many years ago, namely the delivery problem. As these therapeutic platforms are usually taken up by endocytosis, they remain trapped inside the endosome, behind the lipid bilayer, and as such are outside of the cytoplasm and the nucleus. ${ }^{7}$ With a current endosomal escape not exceeding $0.01 \%$, devising new approaches to get such therapeutic platforms into the nucleus and overcome the endosomal escape abyss is the key technological problem to solve before investigating the full potential of such revolutionary therapeutic technologies.
CRISPR has a double delivery problem because two macromolecules are required for a functional therapeutic system: one, a large catalytic Cas9 recombinase that is $\sim 160 \mathrm{kDa}$ and two, a $\sim 150$ nucleotide tracer/sg RNA. ${ }^{1-2}$ Although these components will invariably get somewhat smaller by using a low molecular

Scheme 1. Preparation, cytosolyic delivery and gene editing efficiency of CC-ZIFs. (a) Encapsulating the negatively charged Cas9/sgRNA within positively charged ZIF-8 to form CC-ZIFs. (b) Endosomal escape of CC-ZIFs. (c) CLSM images of cells before and after treatment with CC-ZIFs.

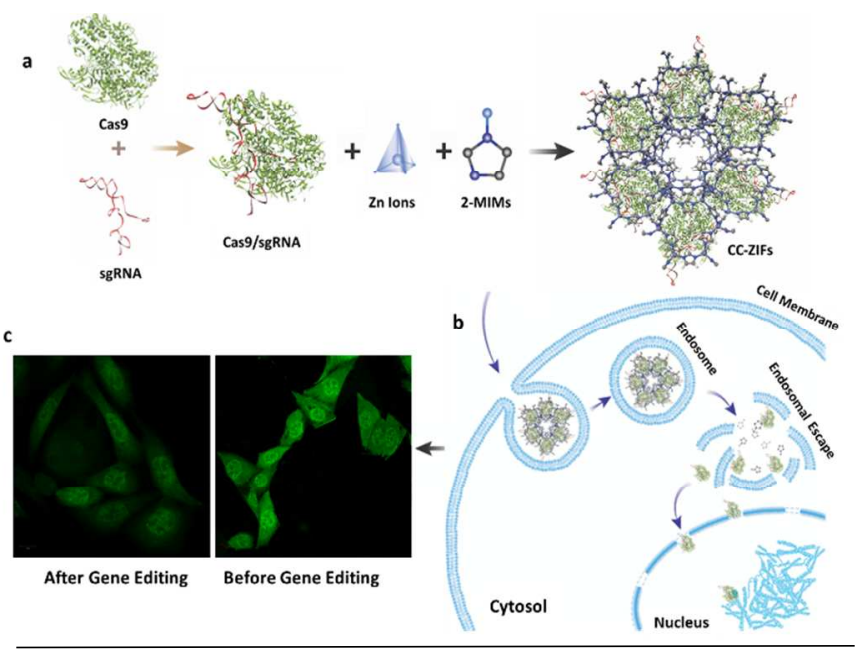

weight CRISPR recombinase and/or shorter sgRNAs, from a delivery perspective, these are still very large and charged problematic macromolecules. Most of CRISPR/Cas9 studies so far have depended on viral or physical delivery; however, the immunogenicity complications of the viral vectors and the difficulty of translating physical delivery protocols in vivo have empowered the need to accomplish such delivery through synthetic nanoparticles. ${ }^{8-9}$ Cationic lipid nanoparticles, ${ }^{10}$ gold nanoparticles ${ }^{11}$ and DNA nanoclews ${ }^{12}$ have been successfully employed for delivery of Cas9. More recently zwitterionic amino lipids were used as delivery vehicles, 
however the co-delivery was of Cas9 mRNA and not the actual protein. ${ }^{13}$

Zeolitic imidazolate framework-8 (ZIF-8) are a subclass of metal-organic frameworks formed by coordination between $\mathrm{Zn}^{2+}$ ions and 2-methylimidazole. ${ }^{14-16}$ They have good biocompatibility $^{17,18}$ and remarkably tunable pore openings ${ }^{19}$ for a wide range of guest molecules. Recently, ZIFs have been successfully used for drug delivery ${ }^{20}$, ATP imaging ${ }^{21}$ and biomimetic mineralization or sheltering of biomacromolecules. $^{22,23}$ Moreover, the imidazole linkers are known to have excellent $\mathrm{pH}$-buffering capacity, which is presumed to confer enhanced ability to escape the endocytic pathway. ${ }^{24}$, 25 Herein, we report the first example of nanoscale ZIF-8 coencapsulating Cas9 protein and sgRNA (CC-ZIFs) with a remarkable loading efficiency of $17 \%$ and enhanced endosomal escape leading to $37 \%$ reduction in gene expression over four days (Scheme 1). This work opens up new possibilities for exceptionally improved genetic materials delivery enabled by precisely engineered biocompatible smart coordination frameworks.

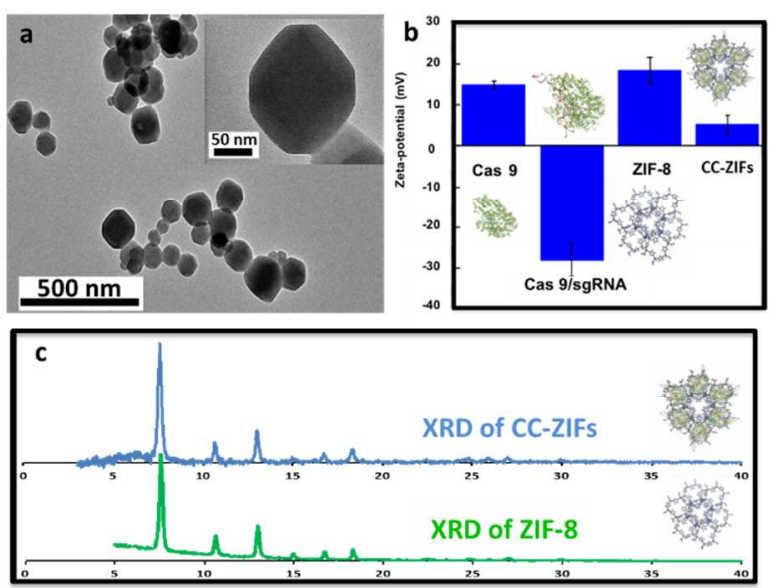

Figure 1. Characterization of CC-ZIFs. (a) TEM micrographs of CC-ZIFs at different scales. (b) Zeta potential of free Cas9, Cas9/sgRNA complex, ZIF-8 and CC-ZIFs. Bars represent mean \pm SD $(n=3)$. (c) PXRD of ZIF-8 (green) and CC-ZIFs (blue).

CC-ZIFs were prepared by first mixing Cas9 and sgRNA at a molar ratio of 1:1 in a phosphate buffer saline (PBS) for 5 minutes followed by addition of 2-methylimidazole solution (o.9 mL, $5 \mathrm{M}, \mathrm{pH}_{7}$ ). An aqueous solution of zinc nitrate $(0.1$ $\mathrm{mL}, 0.5 \mathrm{M}$ ) was then slowly added at room temperature under mechanical agitation for 20 minutes after which the clear solution turned opaque (Scheme 1a). The resulting CC-ZIFs were then collected by centrifugation after washing with DI water (3 cycles) to remove all residues. The truncated ZIF- 8 cubic crystals were first characterized by tunneling electron microscopy (TEM) showing an average size of $100 \mathrm{~nm}$ that is ideal for intracellular delivery (Figure 1a). The average size was confirmed by dynamic light scattering analysis (DLS) at neutral conditions (Figure S1). Measuring zeta potential showed that the positively charged Cas9 $(+15 \mathrm{mV} \pm 1)$ became negatively charged upon the addition of sgRNA $(-28 \mathrm{mV} \pm 4)$. After metal-coordination sheltering of the complex and formation of CC-ZIFs the zeta potential measured at $+5 \mathrm{mV} \pm 2$, which is less positive than the native ZIF-8 $(+17 \mathrm{mV} \pm 1$ ) (Figure $1 \mathrm{~b})$. Furthermore, powder $\mathrm{X}$ - ray diffraction (PXRD) graphs showed that the Cas9/sgRNA@ZIF-8 has the same crystal structure as that of the pure ZIF-8 (Figure 1c).
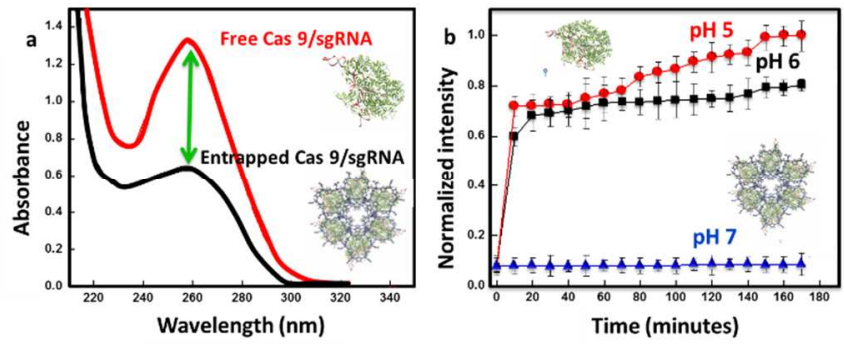

Figure 2. CRISPR/Cas9 loading and release. (a) UV-VIS spectra of Alexa fluora (AF) labeled CC-ZIFs compared to free AFCas9/sgRNA complex. (b) $\mathrm{pH}$ dependent release of AFCas9/sgRNA from CC-ZIFs.

CRISPR/Cas9 loading efficiency and $\mathrm{pH}$ responsive release were tested by labeling Cas9 with Alexa fluor 647 (AF). The red fluorescence of AF-Cas9 in the supernatant decreased drastically after the de novo encapsulation within ZIF-8 (Figure S2a). The loading capacity was calculated to be 1.2 wt \% with efficiency of $17 \%$ (Figure $2 \mathrm{a}$ ), which is consistent with the loading capacity of previously reported MOF based delivery systems. ${ }^{23}$ To evaluate the stability of AF-CC-ZIFs under physiological ( $\mathrm{pH} 7.4$ ) and acidic ( $\mathrm{pH}_{5}$ and 6) conditions, they were dispersed in PBS solution. DLS analysis showed a stable structure at neutral conditions that starts degrading directly at acidic $\mathrm{pH}$ (Figure $\mathrm{S}_{1}$ ). CC-ZIFs completely decomposed after 6 hours at $\mathrm{pH}_{5.5}$ (Figure $\mathrm{S}_{1}$ ). The release of AFCas9 was monitored by fluorescence spectroscopy (Figure $2 \mathrm{~b}$, Figure S2b). Under physiological conditions less than $3 \%$ of AF-Cas9 was released while $60 \%$ and $70 \%$ were released in 10 minutes at $\mathrm{pH} 6$ and 5 , respectively. The maximum release of AF-Cas9 was observed at $\mathrm{pH} 5$ within 3 hours, exploiting the selectivity of CC-ZIFs release under acidic conditions. The release mechanism of CC-ZIFs is superior to reported protein delivery systems, such as Au NPs and DNA cages, in a sense that it is associated with a complete and safe dissolution of ZIF-8 at low $\mathrm{pH}$ without leaving residue nanoparticles or denaturation of DNA strands.

Biocompatibility of CC-ZIFs was tested at different concentrations by incubation with Chinese hamster ovary (CHO) cells for 12 hours. $\mathrm{CHO}$ cells were washed and evaluated by the cell counting kit-8 (CCK-8). Compared to native $\mathrm{CHO}$ group, no measurable impact was seen at concentrations below 200 $\mu \mathrm{g} \mathrm{mL}{ }^{-1}$, whereas higher concentrations $\left(\geq 200 \mu \mathrm{g} \mathrm{mL}^{-1}\right)$ led to the increased cytotoxicity in both ZIF-8 and CC-ZIFs (Figure $\left.\mathrm{S}_{3} \mathrm{a}, \mathrm{S}_{3} \mathrm{~b}\right)$. Based on the CCK-8 results, we examined the toxicity of 250 and $100 \mu \mathrm{gL} \mathrm{mL}^{-1}$ of ZIF- 8 and CC-ZIFs by live-dead cell staining using flow cytometry (Figure $\mathrm{S}_{3} \mathrm{c}-\mathrm{S}_{3} \mathrm{f}$ ). No significant cell death was observed even after 12 hours of transfection at a concentration of $100 \mu \mathrm{g} \mathrm{mL}^{-1}$. The lethal dose (LD5o) of ZIFs and CC-ZIFs was determined to be 400 and $420 \mu \mathrm{g}$ $\mathrm{mL}^{-1}$, respectively (Figure $\mathrm{S}_{3} \mathrm{~g}$ ). Based on these results, a concentration of $100 \mu \mathrm{g} \mathrm{mL}^{-1}$ of CC-ZIFs was used in all subsequent experiments. We examined the capability of ZIF-8 to deliver AF-Cas9/sgRNA by flow cytometry and confocal laser scanning microscopy (CLSM). The intensity of red AF 647 
increased with time suggesting the delivery of more Cas9/sgRNA into the cytoplasm (Figure S4a). CLSM images showed that AF-Cas9/sgRNA internalized in the nucleus after 3 hours (Figure S4b). In 6 hours, many AF-Cas9/sgRNA were seen in nuclei, elucidating the increase in the AF-Cas9 signal in the flow cytometry data (Figure $\mathrm{S}_{4} \mathrm{~b}$ ). As a control, free AF-Cas9/sgRNA was not delivered into cells as the signal of AF-Cas9/sgRNA was not detected by flow cytometry (Figure $\mathrm{S}_{4 \mathrm{c}}$ ) or CLSM (Figure $\mathrm{S}_{4} \mathrm{~d}$ ) over the same period of time.

$1 \mathrm{~h}$
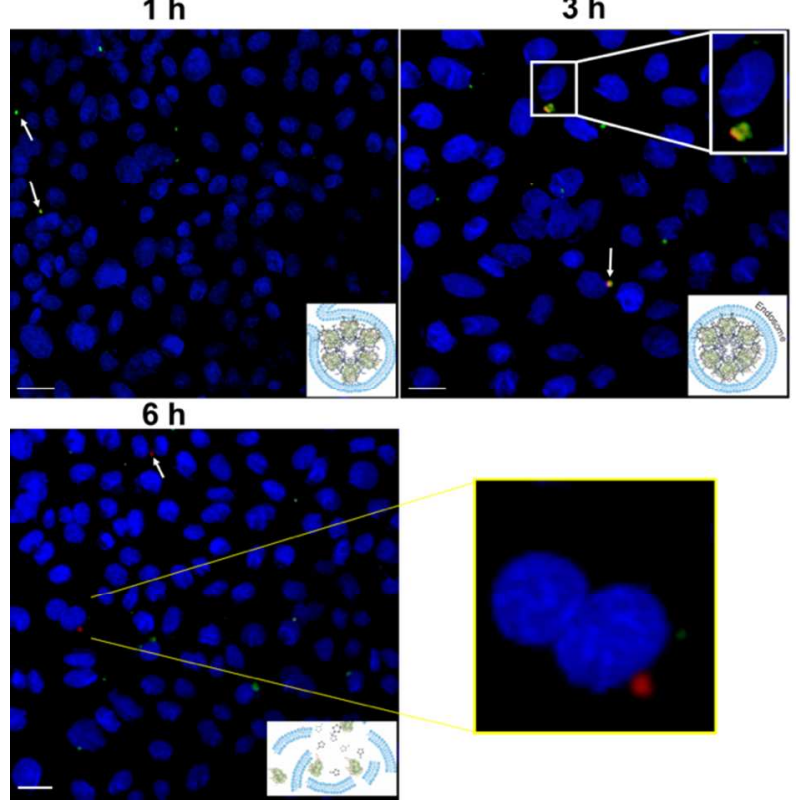

Figure 3. CLSM images (40x) of CHO cells after incubation with AF-CC-ZIFs (red) for 1, 3 and 6 hours. Lysotracker Green (green) was used to stain the acidic organelles (endosomes). The merged images are used to confirm that AF-Cas9/sgRNA is released and delivered into the nucleus within 6 hours of uptake. Scar bar: $20 \mu \mathrm{m}$.

To assess the endosomal escape associated with CC-ZIFs, $\mathrm{CHO}$ cells were first transfected with particles at different time points (1, 3 and 6 hours), to examine the cellular transfection efficiency. The endosomal escape of CC-ZIFs is mediated by the protonation of the imidazole ring, ${ }^{24}$ which is followed by the release of Cas9/sgRNA to the cytoplasm. AFCas9/sgRNA localized inside endosomes within 1 hour of transfection, which was observed in yellow merged color (Figure 3). In contrast, 3 and 6 hours post transfection showed complete separation between red Cas9 and green endosomes indicating the release of Cas9/sgRNA from endosomes. Images at 2ox were also obtained to show that many cas9 were released form ZIF-8 upon their protonation (Figure S6). We then compared our system's ability to escape endosomal encapsulation to a cationic liposome based delivery system. ${ }^{10}$ The obtained results with the same cell line over the same period of time showed a complete localization of the liposomes within endosomes (Figure $\mathrm{S}_{5}$ ).

To demonstrate the efficiency of gene knockdown by CCZIFs, we used sgRNA to target the coding region of the enhanced green fluorescent protein (EGFP) in CHO cells. Targeting the coding region will result in shifting the reading frame and consequently preventing proper EGFP expression.
EGFP transfected $\mathrm{CHO}$ cells were treated with free Cas9/sgRNA, ZIF-8 and CC-ZIFs for 2 days. We also investigated whether incubating cells for more than 2 days would change EGFP gene expression so cells were incubated for 4 days after treatment. The fluorescence and expression of EGFP were determined by CLSM, flow cytometry and quantitative real-time polymerase chain reaction (qRT-PCR). Measuring the EGFP fluorescence using flow cytometry demonstrates that CC-ZIFs reduced the fluorescence of EGFP by $31 \%$ when incubated for 2 days and $37 \%$ when incubated for 4 days (Figure 4a, Figure 4c).
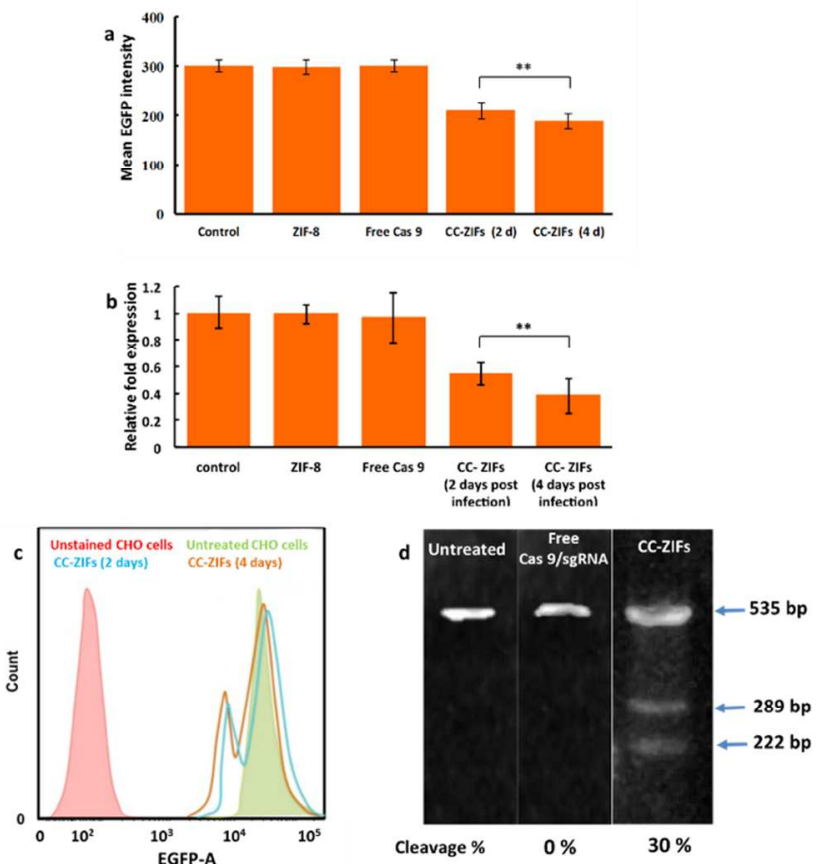

Figure 4. Genome editing by CC-ZIFs. (a, c) Flow cytometry analysis of EGFP expression at day 2 and 4 at concentration of $100 \mu \mathrm{g} \mathrm{mL}^{-1}$ (encapsulated Cas9/sgRNA $240 \mathrm{nM}$ ). (b) quantitation of EGFP expression by qPCR at concentration of 240 $\mathrm{nM}$ for 2 and 4 days. Statistical analysis was determined using unpaired t-test $\left({ }^{* *} \mathrm{P}<0.001,{ }^{*} \mathrm{P}<0.01,{ }^{*} \mathrm{P}<0.1\right)$. (d) Surveyor assay for indel frequency analysis of EGFP transfected CHO cells incubated with free Cas9/sgRNA and CC-ZIFs.

No effect was seen on the EGFP fluorescence when cells were treated with free Cas9/sgRNA and ZIF-8, demonstrating the importance of ZIF-8 in the delivery of Cas9/sgRNA. CLSM images also confirmed the quenching of the EGFP fluorescence when ZIF-8 was used to deliver Cas9/sgRNA (Figure $\mathrm{S}_{7}$ ). These results were refined by quantifying the mRNA levels of EGFP in free Cas9/sgRNA, ZIF-8 and CC-ZIFs treated $\mathrm{CHO}$ cells by qRT-PCR. Our results demonstrate that CCZIFs effectively delivered Cas9/sgRNA and the sgRNA targeted the EGFP coding region causing 2-fold repression of EGFP gene when cells were incubated for 2 days (Figure $4 \mathrm{~b}$ ). Incubating cells for 4 days post treatment resulted in decreasing the fluorescence of EGFP by $37 \%$ and reduction of the level of EGFP gene expression by 3-folds. Free Cas9/sgRNA and ZIF-8 had no effect on the expression of EGFP (Figure $4 \mathrm{~b}$ ). The efficiency of gene knockdown by CC-ZIFs was compared to the Lipofectamine CRISPRMAX Cas9. The concentration 
of Cas9/sgRNA used in lipofectamine was $240 \mathrm{nM}$. The Lipofectamine CRISPRMAX Cas9 resulted in only1-fold and 1.5-fold repression of EGFP gene in 2 and 4 days after treatment, respectively (Figure S8). Surveyor assay revealed mutation frequencies of o $\%$ and $30 \%$ for cells treated with free Cas9/sgRNA and CC-ZIFs for 2 days (Figure 4c), respectively, closely paralleling the results of flow cytometry, CLSM and qRT-PCR.

In conclusion, we demonstrate the first example of CRISPR/ Cas9 delivery by metal-coordination frameworks. ZIF-8 can successfully shelter both the large Cas9 protein and the negatively charged sgRNA with a high loading capacity of $1.2 \mathrm{wt} \%$. The protonation of the imidazole based framework at endosomal $\mathrm{pH}$ promotes fast endosomal escape and enhanced nuclei delivery, which is paramount for genetic transfection. This system provides a one-step route for the creation of effective and transient gene editing technology.

\section{ASSOCIATED CONTENT}

\section{Supporting Information}

The Supporting Information is available free of charge on the ACS Publications website.

Characterization data (DLS, TEM and fluorescence spectroscopy), Cytotoxicity and uptake data.

\section{AUTHOR INFORMATION}

\section{Corresponding Author \\ * Niveen.khashab@kaust.edu.sa \\ REFERENCES}

1. Jinek, M.; Chylinski, K.; Fonfara, I.; Hauer, M.; Doudna, J. A.; Charpentier, E., A programmable dual-RNA-guided DNA endonuclease in adaptive bacterial immunity. Science 2012, 337 (6096), 816-21.

2. Cong, L.; Ran, F. A.; Cox, D.; Lin, S. L.; Barretto, R.; Habib, N.; Hsu, P. D.; Wu, X. B.; Jiang, W. Y.; Marraffini, L. A.; Zhang, F., Multiplex Genome Engineering Using CRISPR/Cas Systems. Science 2013, 339 (6121), 819-823.

3. Mali, P.; Yang, L.; Esvelt, K. M.; Aach, J.; Guell, M.; DiCarlo, J. E.; Norville, J. E.; Church, G. M., RNA-guided human genome engineering via Cas9. Science 2013, 339 (6121), 823-6.

4. Liao, H. K.; Gu, Y.; Diaz, A.; Marlett, J.; Takahashi, Y.; Li, M.; Suzuki, K.; Xu, R.; Hishida, T.; Chang, C. J.; Esteban, C. R.; Young, J.; Belmonte, J. C. I., Use of the CRISPR/Cas9 system as an intracellular defense against HIV-1 infection in human cells. Nature Communications 2015, 6.

5. Choudhary, E.; Thakur, P.; Pareek, M.; Agarwal, N., Gene silencing by CRISPR interference in mycobacteria. Nat Commun 2015, 6, 6267. 6. Hemphill, J.; Borchardt, E. K.; Brown, K.; Asokan, A.; Deiters, A., Optical Control of CRISPR/Cas9 Gene Editing. J Am Chem Soc 2015, 137 (17), 5642-5

7. Dowdy, S. F., Overcoming cellular barriers for RNA therapeutics. Nat Biotechnol 2017, 35 (3), 222-229.

8. Mout, R.; Ray, M.; Lee, Y. W.; Scaletti, F.; Rotello, V. M., In Vivo Delivery of CRISPR/Cas9 for Therapeutic Gene Editing: Progress and Challenges. Bioconjug Chem 2017, 28 (4), 880-884.

9. Wang, H. X.; Li, M.; Lee, C. M.; Chakraborty, S.; Kim, H. W.; Bao, G.; Leong, K. W., CRISPR/Cas9-Based Genome Editing for Disease Modeling and Therapy: Challenges and Opportunities for Nonviral Delivery. Chem Rev 2017, 117 (15), 9874-9906.
10. Zuris, J. A.; Thompson, D. B.; Shu, Y.; Guilinger, J. P.; Bessen, J. L.; Hu, J. H.; Maeder, M. L.; Joung, J. K.; Chen, Z. Y.; Liu, D. R., Cationic lipid-mediated delivery of proteins enables efficient protein-based genome editing in vitro and in vivo. Nat Biotechnol 2015, 33 (1), 7380.

11. Mout, R.; Ray, M.; Yesilbag Tonga, G.; Lee, Y. W.; Tay, T.; Sasaki, K.; Rotello, V. M., Direct Cytosolic Delivery of CRISPR/Cas9Ribonucleoprotein for Efficient Gene Editing. ACS Nano 2017, 11 (3), 2452-2458.

12. Sun, W.; Ji, W.; Hall, J. M.; Hu, Q.; Wang, C.; Beisel, C. L.; Gu, Z., Self-assembled DNA nanoclews for the efficient delivery of CRISPRCas9 for genome editing. Angew Chem Int Ed Engl 2015, 54 (41), 12029-33.

13. Miller, J. B.; Zhang, S.; Kos, P.; Xiong, H.; Zhou, K.; Perelman, S. S.; Zhu, H.; Siegwart, D. J., Non-Viral CRISPR/Cas Gene Editing In Vitro and In Vivo Enabled by Synthetic Nanoparticle Co-Delivery of Cas9 mRNA and sgRNA. Angew Chem Int Ed Engl 2017, 56 (4), 10591063.

14. Li, H. E., M.; O'Keeffe, M.; Yaghi, O. M., Design and synthesis of an exceptionally stable and highly porous metal-organic framework Nature 1999, 402 (6759), 276-279.

15. Phan, A.; Doonan, C. J.; Uribe-Romo, F. J.; Knobler, C. B.; O'Keeffe, M.; Yaghi, O. M., Synthesis, structure, and carbon dioxide capture properties of zeolitic imidazolate frameworks. Acc Chem Res 2010, $43(1), 58-67$.

16. Venna, S. R.; Carreon, M. A., Highly permeable zeolite imidazolate framework-8 membranes for $\mathrm{CO}_{2} / \mathrm{CH}_{4}$ separation. J Am Chem Soc 2010, 132 (1), 76-8.

17. Doonan, C.; Ricco, R.; Liang, K.; Bradshaw, D.; Falcaro, P., MetalOrganic Frameworks at the Biointerface: Synthetic Strategies and Applications. Accounts Chem Res 2017, 50 (6), 1423-1432.

18. Horcajada, P.; Gref, R.; Baati, T.; Allan, P. K.; Maurin, G.; Couvreur, P.; Ferey, G.; Morris, R. E.; Serre, C., Metal-Organic Frameworks in Biomedicine. Chemical Reviews 2012, 112 (2), 12321268.

19. Yang, J.; Zhang, Y. B.; Liu, Q.; Trickett, C. A.; Gutierrez-Puebla, E.; Monge, M. A.; Cong, H.; Aldossary, A.; Deng, H.; Yaghi, O. M., Principles of Designing Extra-Large Pore Openings and Cages in Zeolitic Imidazolate Frameworks. J Am Chem Soc 2017, 139 (18), 6448-6455.

20. Adhikari, C.; Das, A.; Chakraborty, A., Zeolitic Imidazole Framework (ZIF) Nanospheres for Easy Encapsulation and Controlled Release of an Anticancer Drug Doxorubicin under Different External Stimuli: A Way toward Smart Drug Delivery System. Mol Pharm 2015, 12 (9), 3158-66.

21. Deng, J.; Wang, K.; Wang, M.; Yu, P.; Mao, L., Mitochondria Targeted Nanoscale Zeolitic Imidazole Framework-9o for ATP Imaging in Live Cells. J Am Chem Soc 2017, 139 (16), 5877-5882.

22. Liang, K.; Ricco, R.; Doherty, C. M.; Styles, M. J.; Bell, S.; Kirby, N.; Mudie, S.; Haylock, D.; Hill, A. J.; Doonan, C. J.; Falcaro, P., Biomimetic mineralization of metal-organic frameworks as protective coatings for biomacromolecules. Nature Communications 2015, 6 .

23. Shieh, F. K.; Wang, S. C.; Yen, C. I.; Wu, C. C.; Dutta, S.; Chou, L. Y.; Morabito, J. V.; Hu, P.; Hsu, M. H.; Wu, K. C. W.; Tsung, C. K., Imparting Functionality to Biocatalysts via Embedding Enzymes into Nanoporous Materials by a de Novo Approach: Size-Selective Sheltering of Catalase in Metal-Organic Framework Microcrystals. Journal of the American Chemical Society 2015, 137 (13), 4276-4279. 24. Mishra, S.; Heidel, J. D.; Webster, P.; Davis, M. E., Imidazole groups on a linear, cyclodextrin-containing polycation produce enhanced gene delivery via multiple processes. J Control Release 2006, 116 (2), 179-91.

25. Li, M.; Tao, Y.; Shu, Y. L.; LaRochelle, J. R.; Steinauer, A.; Thompson, D.; Schepartz, A.; Chen, Z. Y.; Liu, D. R., Discovery and Characterization of a Peptide That Enhances Endosomal Escape of Delivered Proteins in Vitro and in Vivo. Journal of the American Chemical Society 2015, 137 (44), 14084-14093. 


\section{TOC Figure}

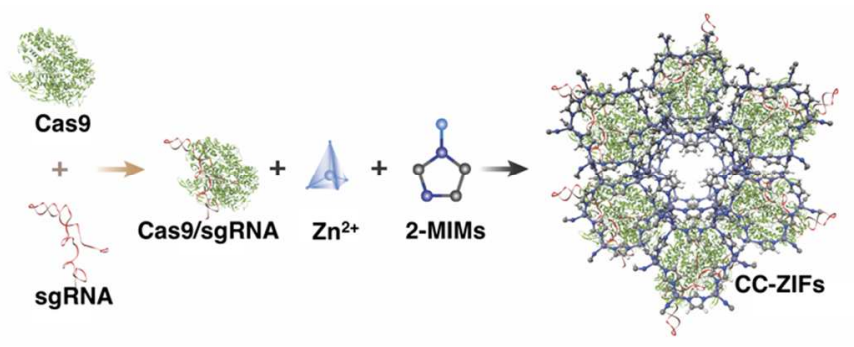

57 